\title{
ON ELEMENTAL ANNIHILATOR RINGS
}

\author{
by R. YUE CHI MING
}

(Received 22nd September 1969)

\section{Introduction}

Throughout this note $A$ denotes a ring with identity, and " module " means "left unitary module". In (2), C. Yohe studied elemental annihilator rings (e.a.r. for brevity). An e.a.r. is defined as a ring in which every ideal is the annihilator of an element of the ring. For example, a semi-simple, Artinian ring is an e.a.r. $A$ is a l.e.a.r. (left elemental annihilator ring) if every left ideal is the left annihilator of an element of the ring. A r.e.a.r. (right elemental annihilator ring) is defined similarly.

C. R. Yohe has proved (2, Theorem II): Let $A$ be a semi-prime ring. Then the following properties are equivalent:

(i) $A$ is both a l.e.a.r. and a r.e.a.r.

(ii) $A$ is a l.e.a.r., and has ascending chain condition on right annihilators.

(iii) $A$ is a direct sum of matrix rings over division rings.

This short note answers the following question raised in (2, p. 1348): what are the consequences of removing the e.a.r. condition from one side in the statement of the above theorem? We prove that the theorem is valid with the e.a.r. condition on one side only. We also prove that a regular 1.e.a.r. is semi-simple Artinian.

Let us recall:

(a) A submodule $N$ is essential in a module $M$ if, for any submodule $\boldsymbol{P}$ of $M, N \cap P=0$ implies $P=0$. We write $L(M)$ for the lattice of essential left $A$-submodules of $M$.

(b) $Z(A)=\{x \in A \mid$ the left annihilator $(0: x)$ is essential in $A\}$ is the (left) singular ideal of $A$.

(c) $A$ is semi-simple Artinian if and only if every $A$-module is injective. (See, for example (1).)

We say that $A$ is a "l.s.a.r." if every essential left ideal of $A$ is the annihilator of some subset of $A$. Thus a l.e.a.r. is a 1.s.a.r.

Theorem 1. Let $A$ be a l.s.a.r. with $Z(A)=0$. Then $A$ is semi-simple Artinian.

Proof. Let $M$ be an arbitrary module with injective hull $E$. For $x \in E$, $(M: x) \in L(A)$. For if $J$ is a non-zero left ideal of $A$, then either $J x=0$, which implies $J \subseteq(M: x) \cap J$, or $J x \neq 0$, in which case $M \cap J x \neq 0$. Therefore 
$(M: x) \cap J \neq 0$ which proves that $(M: x) \in L(A)$. By hypothesis, $(M: x)$ is the left annihilator of some subset $S$ of $A$. Thus $(M: x)=(0: S)$. If $t \in S$, then $(M: x) \leqq(0: t)$ which shows $t \in Z(A)=0$. Hence $S=0$ which proves that $x \in M$ and $M$ is therefore injective. $A$ is therefore semi-simple Artinian.

Corollary. A (von Neumann) regular, l.e.a.r. is semi-simple Artinian.

Proof. Since $A$ is regular, $Z(A)=0$. We now prove our main result.

Theorem 2. If $A$ is a semi-prime l.s.a.r., then $A$ is semi-simple Artinian.

Proof. It is sufficient to show that $Z(A)=0$. Theorem 1 then applies. We assume $Z(A) \neq 0$ and derive a contradiction. Let $I$ be maximal in the family of left ideals whose intersection with $Z(A)$ is zero. Then $Z(A) \oplus I \in L(A)$. Now $Z(A) \oplus I=(0: T)$ for some subset $T$ of $A$. If $T=0, Z(A) \oplus I=A$. Since $Z(A)$ is a direct summand of $A, Z(A)=A e$, where $e^{2}=e$. Then $(0: e) \cap A e=0$ which contradicts $e \in Z(A)$. Hence $T \neq 0$. If $0 \neq w \in T$, since $(Z(A) \oplus I) \subseteq(0: w), w \in Z(A)$ which implies

$$
(A w)^{2} \subseteq Z(A) \cdot A w=Z(A) \cdot w \subseteq(Z(A) \oplus I) \cdot w=0,
$$

which contradicts $A$ semi-prime. This proves Theorem 2 .

Corollary. A semi-prime, l.e.a.r. is semi-simple Artinian (see (2, Theorem 2)).

Remark. If $A$ is a l.e.a.r. and r.e.a.r., then $A$ is a principal ideal ring in which every regular element (i.e. every non-zero-divisor) is invertible.

Proof. The fact that $A$ is principal is implicit in the proof of $(2$, Theorem 1$)$. Let $c$ be a regular element of $A$. Then $A c=(0: b)$, some $b \in A$. Since $c b=0$, $b=0$ which proves $A c=A$. Similarly, $c A=A$ which proves $c$ is invertible.

\section{Acknowledgements}

We are indebted to Professor G. Rinehart for helpful comments.

We would also like to thank the referee for helpful suggestions.

Note added in proof

This problem has also been considered by J. K. Luedemann, Math. Ann. 185 (1970), 309-314.

\section{REFERENCES}

(1) C. FAITH, Lectures on injective modules and quotient rings, Lecture notes in Mathematics no. 49 (Springer-Verlag, Berlin-Heidelberg-New York, 1967).

(2) C. R. YoHE, On rings in which every ideal is the annihilator of an element, Proc. Amer. Math. Soc. 19 (1968), 1346-1348.

\section{BhujoharRy COLLEge}

St George Street

PORT Louis, MaUritius 